\title{
Oxyures de Muridae africains
}

\author{
Par Jean-Claude QUENTIN
}

Les Oxyures de Muridae éthiopiens sont très peu connus. Une seule espèce, Syphacia (Syphacia) nigeriana Baylis 1928, parasite de Mastomys erythroleucus (Temm.) a jusqu'à présent été décrite.

Bon nombre de Muridae, capturés en plusieurs localités d'Afrique Equatoriale et aimablement communiqués par M. F. Petter, hébergent au niveau du caecum et du rectum de nombreux Oxyures. Ceux-ci appartiennent à cinq espèces distinctes:

- Deux sont communes et cosmopolites chez les Muridae. Ce sont: Syphacia (Syphacia) obveolata (Rud. 1802) et Aspiculuris tetraptera (Nitzsh 1821).

- Trois nous semblent nouvelles: Syphacia (Syphacia) lophuromyos n. sp. parasite de Lophuromys sikapusi Temm., Syphacia (Syphacia) megaloon n. sp. parasite de Mus minutoides (Smith) et $M$. setulosus (Peters), et Aspiculuris africana n. sp. très répandu chez Mastomys sp. à 32 chromosomes, Praomys jacksoni (de Winton) et Thamnomys rutilans (Peters).

\section{SYPHACIA (SYPHACIA) OBVEOLATA (Rud. 1802)}

HôTes : un Stochomys longicaudatus Tullberg, deux Praomys jacksoni (de Winton).

Localité: Originaires de Bangui. Autopsiés au laboratoire après un séjour d'un mois au Muséum.

Dates de RÉCOLte : 5-XII-1963, 13-XII-1963, 7-I-1964.

MATÉRIEL ÉTUdí́ : 40 \% et $10 \sigma^{\circ}$.

Les dimensions des mâles et des femelles qui présentent deux ailes latérales très étroites correspondent à celles de l'espèce Syphacia $(S$.) obveolata (Rud. 1802) données par Seurat (1916) et Roman (1951).

La distance de l'anus à l'extrémité caudale de la femelle, qui est un des principaux caractères séparant l'espèce $S$. $(S$.) obveolata où cette dimension est de $530-675 \mu$ (Roman 1951) de l'espèce $S$. (S.) nigeriana Baylis 1928 où la longueur correspondante est 850-950 $\mu$, mesure sur notre matériel 550-600 $\mu$. Chez les mâles, la distance anuspointe caudale, qui est de 120 à $140 \mu$ d'après Roman, est légèrement inférieure sur nos spécimens, où elle atteint $110 \mu$. 
La principale différence relevée avec les descriptions de Seurat et de Roman concerne la longueur des œufs : $100 \mu$ sur notre matériel, 115 d'après Seurat, 118$153 \mu$ chez les femelles de $S$. ( $S$.) obveolata récoltées par Roman.

Nous pensons que ces différences ne justifient pas la création d'une espèce nouvelle et identifions notre matériel à $S$. (S.) obveolata (Rud. 1802).

\section{SYPHACIA (SYPHACIA) LOPHUROMYOS n. sp.}

HôTE : Lophuromys sikapusi Temm.

LOCALITÉ : Boukoko.

DATES DE RÉCOLTE : 23-X-1963, 30-X-1963, 20-XI-1964 ( $९$ allotype) ; 4-XII-1964 (రో holotype).

MatéRIEL ÉtUdí́ : Nombreuses femelles jeunes et gravides et quatre mâles; nous n’avons jamais récolté dè mâles en compagnie de femelles gravides.

\section{Description}

Les mâles et les femelles ne possèdent pas d'ailes latérales. La cuticule est légèrement décollée au niveau céphalique sur une longueur de $300 \mu \mathrm{chez}$ les femelles et de $170 \mu$ chez les mâles. La principale caractéristique de ce Syphacia réside dans la morphologie de son plateau facial. Il est ovale et régulier chez les mâles et les jeunes femelles. Deux papilles submédianes et une amphide sont groupées de chaque côté latéralement et font saillie en avant de la bouche. Un cycle interne de six papilles entoure directement le bord buccal. Trois grandes lèvres: deux latéro-ventrales et une dorsale protègent chacune une dent pharyngienne. Chez les femelles gravides, la bouche s'invagine entraînant une déformation du p'ateau facial.

Mâle (fig. 1 B): Spécimen holotype long de $1.330 \mu$, large de $115 \mu$. Longueur de l'œsophage et du bulbe : $200 \mu$, diamètre du bulbe : $60 \mu$. Anneau nerveux et pore excréteur situés respectivement à 100 et $300 \mu$ de l'apex. Trois bosses cuticulaires, dont les parties moyennes sont à 430,475 et $700 \mu$ de l'apex. Queue longue de $240 \mu$ avec une pointe caudale très fine et très longue de $190 \mu$ (fig. $1 \mathrm{~L}$ ). Le spicule mesure $75 \times$ $5 \mu$. Le gubernaculum en forme de lame est long de $30 \mu$, large de $6 \mu$.

Femelle: De petite taille, la femelle allotype mesure $2.630 \mu$ de long sur $370 \mu$ de large. L'œsophage et le bulbe mesurent $310 \mu$. Diamètre du bulbe : $90 \mu$. Anneau nerveux, pore excréteur et vulve situés respectivement à 130,330 et $550 \mu$ de l'apex. Les dimensions des œufs sont $110 \times 48 \mu$. Queue longue de $410 \mu$.

\section{Discussion}

Les caractères morphologiques les plus intéressants pour la systématique des Syphacia : région céphalique, appareil excréteur et crganes génitaux (Roman 1951), sont, chez notre matériel, différents de ceux des Syphacia cosmopolites de Rongeurs décrits 




Fig. 1. - Syphacia (Syphacia) lophuromyos n. sp. A) Femelle, vue latérale. B) Mâle, vue latérale. C) Tête de jeune femelle, vue apicale. D) Tête de mâle, vue apicale, E, F, G) Différents aspects du plateau facial chez des femelles gravides. H) Tête d'une femelle gravide, vue ventrale. I) Pore excréteur mâle. J, K) Pores excréteurs femelles. L) Extrémité caudale mâle holotype. M) Spicule et gubernaculum. N) Extrémité caudale mâle, vue ventrale. O) Dernière bosse cuticulaire. P) (Euf A : éch. $500_{\mu}$; B : éch. $200_{\mu}$; C, D, E; F, G, H, I, J, K; M, N, O : éch. $50_{\mu} ; \mathrm{L}, \mathrm{P}:$ éch $100_{u}$ Annales de Parasitologie humaine et comparée (Paris), t. 41, 1966, $\mathrm{n}^{\circ} 5$ 
par Roman. Sur nos spécimens, le plateau facial garni de deux cycles de papilles devient dissymétrique du fait de l'invagination de la bouche (fig. $1 \mathrm{C}, 1 \mathrm{D}, 1 \mathrm{E}, 1 \mathrm{~F}, 1 \mathrm{G}$ ). Le pore excréteur est, en vue ventrale, entouré d'une vésicule de contours losangiques, soutenue par des fibres radiées (fig. $1 \mathrm{I}, 1 \mathrm{~J}, 1 \mathrm{~K}$ ). Le spicule chez le mâle n'atteint que $75 \mu$. La longueur de la pointe caudale, par contre, est relativement importante par rapport à la taille du mâle.

Par sa localisation géographique, notre espèce peut être comparée à $S$. ( $S$.) nigeriana Baylis 1928 (1), dont le mâle est de longueur comparable (1,2-1,45 mm); les femelles que nous avons récoltées sont cependant de taille plus réduite : 2,68 mm contre $5,3 \mathrm{~mm}$ chez $S$. (S.) nigeriana. Le spicule est plus court chez notre espèce que chez $S$. (S.) nigeriana, où il mesure $95 \mu$. La pointe caudale est par contre beaucoup plus longue sur nos spécimens mâles.

En conséquence, nous considérons cette espèce comme nouvelle; compte tenu de l'étroite spécificité parasitaire des Syphacia de Muridae signalée par E. Roman et n'ayant pu mettre en évidence cette espèce chez les autres Muridae de République Centre Africaine que nous avons autopsiés, nous pensons qu'elle peut être propre à l'espèce Lophuromys sikapusi (Temm.) et proposons le nom de Syphacia (Syphacia) lophuromyos n. sp.

\section{SYPHACIA (SYPHACIA) MEGALOON n. sp.}

HôTes : Mus minutoïdes (Smith); Mus setulosus (Peters).

Localité : Tschibati (près de Lwiro), Congo Léopoldville.

DATE DE RÉCOLTE : 22-I-1964.

Matériel Étudié : $5 \%$ et $20^{\circ}$ - dont les types - récoltés au niveau du caecum de Mus minutö̈des, $13 \%$ immatures et $2 \sigma^{\circ}$ dans le caecum de Mus setulosus.

\section{Description}

Oxyures dépourvus d'ailes latérales, cuticule légèrement décollée au niveau de la capsule céphalique. Plateau facial élargi dans le sens latéral (fig. $2 \mathrm{C}$ ). Il est orné d'une grosse papille submédiane à chacun de ses angles. Les amphides sont antérieures aux papilles. De chaque côté du plateau facial, chaque paire de papilles et l'amphide correspondante sont groupées et situées antérieurement par rapport à la bouche (fig. $2 \mathrm{D}$ ).

Mâle (fig. 2 B): Le spécimen holotype mesure 1,36 mm de long, $250 \mu$ de large. L'anneau nerveux, le pore excréteur sont respectivement placés à $80 \mu$ et $220 \mu$ de

(1) Nous remercions le $\mathrm{D}^{r}$ W. G. Inglis qui a bien voulu nous prêter des spécimens paratypes oै et $q$ de Syphacia (Syphacia) nigeriana Baylis 1928. 
l'apex. Ventralement, trois bosses cuticulaires caractéristiques du genre ont leur partie moyenne située à $450,600,900 \mu$ de l'apex. La première bosse est très peu accentuée. La queue est longue de $130 \mu$. La pointe terminale mesure à elle seule $105 \mu$. Six papilles représentées sur les figures $2 \mathrm{H}$ et $2 \mathrm{I}$. Pas d'ailes latérales. Spicule long de $61 \mu$. Gubernaculum long de $31 \mu$ avec un crochet terminal non denté (fig. $2 \mathrm{~J}$ ).

FiG. 2. - Syphacia (Syphacia) megaloon n. sp. A) Femelle, vue latérale. B) Mâle, vue latérale. C) Tête, vue apicale. D) Extrémité antérieure, vue ventrale. E) Pore excréteur. F) Euf. G) Extrémité caudale femelle, vue latérale. $H$ ) Extrémité caudale, mâle, vue latérale. I) Idem, vue ventrale. J) Spicule et gubernaculum

A, B : éch. $500 \mu ;$ C, D, E, J : éch. $50 \mu ; \mathrm{F}, \mathrm{H}, \mathrm{I}$ : éch. $100 \mathrm{u}$; G : éch. $200 \mu$

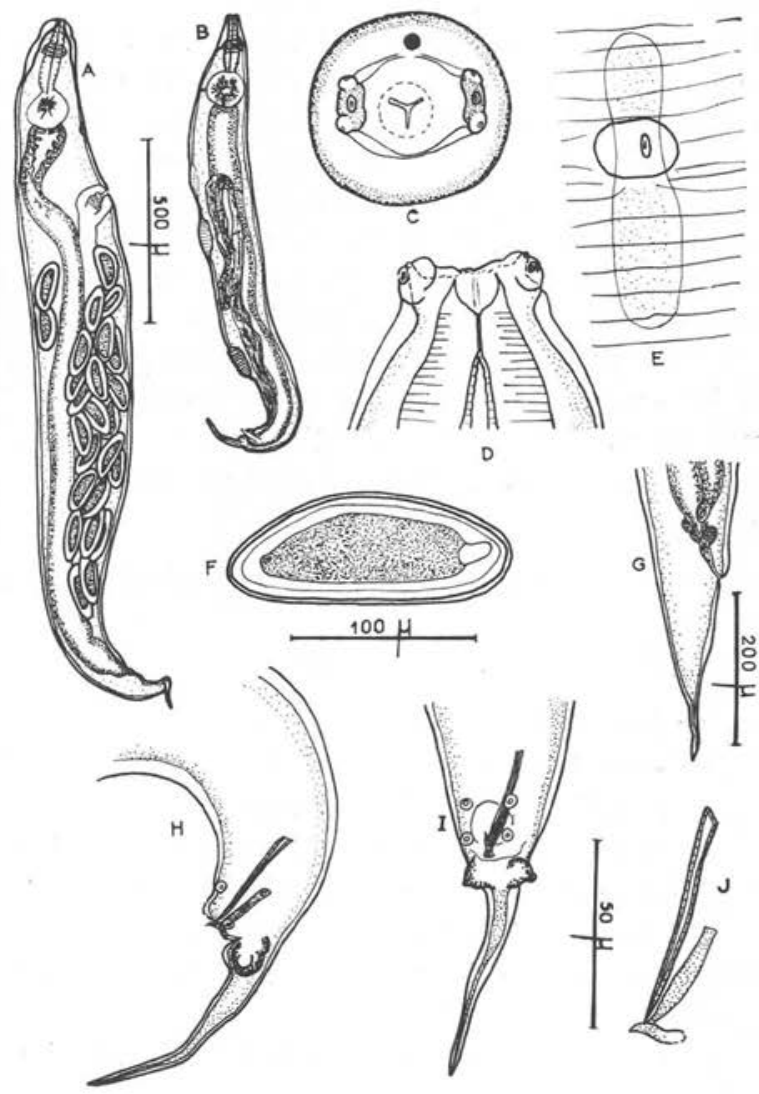

Femelle (fig. 2 A) : Les dimensions de la femelle allotype sont les suivantes: longueur $2 \mathrm{~mm}$, largeur $250 \mu$, œsophage plus bulbe œsophagien $300 \mu$. Anneau nerveux et pore excréteur à 100 et $280 \mu$ de l'apex. Vulve à $480 \mu$ de l'extrémité antérieure. Queue courte mesurant $240 \mu$ (fig. G). Les œufs sont très volumineux et en nombre réduit, environ 20 à 30 pour chaque femelle. Ils présentent la forme caractéristique des œufs de Syphacia, mais leurs très grandes dimensions, $150 \times 60 \mu$, en font un des principaux caractères les distinguant des autres espèces du genre (fig. $2 \mathrm{~F}$ ). 


\section{Discussion}

Notre matériel s'apparente par la taille importante des œufs $(150 \times 60 \mu)$ à l'espèce Syphacia (Syphacia) stroma (Linstow 1884) dont les œufs atteignent 130 à $143 \mu$ de long sur 42 à $52 \mu$ de large d'après Morgan 1932, 123-150 × 41-64 $\mu$ d'après Roman 1951, 137-160 $\mu \times 38-49 \mu$ d'après Bernard 1961. Les mâles que nous avons récoltés sont plus petits $(1,36 \mathrm{~mm})$, que ceux décrits par Morgan $(1,74-2,2 \mathrm{~mm})$ et par Roman (1,45-2,25 mm) mais sont de taille voisine de celle mesurée par Bernard $(1,12$ $1,596 \mathrm{~mm})$. Il faut cependant noter des différences importantes dans la taille du spicule chez le mâle : $61 \mu$ chez nos spécimens et $77 \mu$ chez les mâles de $S$. (S.) stroma décrits par Morgan, 75-90 $\mu$ d'après Roman et 72,2 à 83,3 $\mu$ chez les mâles de $S$. (S.) stroma récoltés par Bernard. En outre, nos spécimens mâles ne possèdent pas d'ailes latérales de part et d'autre de la bourse caudale, alors que Morgan les mentionne nettement pour $S$. (S.) stroma. La taille des femelles enfin est considérablement plus réduite $(2 \mathrm{~mm})$ que celle des spécimens récoltés par Morgan (3,4-3,95 $\mathrm{mm})$, Roman $(3,65-4,85 \mathrm{~mm})$ et Bernard $(3,11-3,78 \mathrm{~mm})$.

Nous pensons donc que notre matériel parasite du caecum de Mus minutoïdes et de $M$. setulosus représente une espèce distincte de $S$. (S.) stroma parasite de l'intestin grêle du mulot Apodemus sylvaticus L. et des espèces voisines. Nous la considérons comme nouvelle et la nommons Syphacia (Syphacia) megaloon n. sp.

\section{ASPICULURIS TETRAPTERA (Nitzsch 1821)}

HôTES, LOCALITÉS, DATES DE RÉCOLTE :

- Mastomys sp. à 32 chromosomes, Bangui, 7-I-1964 ; La Maboké R.C.A., 9-I-1964.

- Praomys jacksoni (de Winton), La Maboké, R.C.A., 25-VIII-1964, 28-VIII-1964.

- P. tullbergi (Thos), Palimé (Togo), 25-I-1965.

- Thamnomys rutilans (Peters), Bébé, R.C.A., 3-XI-1963.

Matériel Étudié : Nombreux spécimens ơ et $q$.

Cet Oxyure cosmopolite, très commun chez les Rongeurs, a été récolté en très grande quantité au niveau du caecum et du rectum des Muridae précités.

\section{ASPICULURIS AFRICANA n. sp.}

Hôtes, LOCALITÉS, DATES DE RÉCOLTE :

- Mastomys sp. à 32 chromosomes, Bébé, R.C.A., 29-X-1963, 5-XII-1963 (types), 6-XII-1963, 2-I-1964.

- Praomys jacksoni (de Winton), Palimé, 28-VIII-1964; Praomys tullbergi (Thos.), Palimé, 25-I-1965.

- Thamnomys rutilans (Peters), Bébé, 27-X-1963.

MatéRIEl ÉTUdié : Nombreux mâles et femelles souvent en association avec Aspiculuris tetraptera (Nitzsch). 


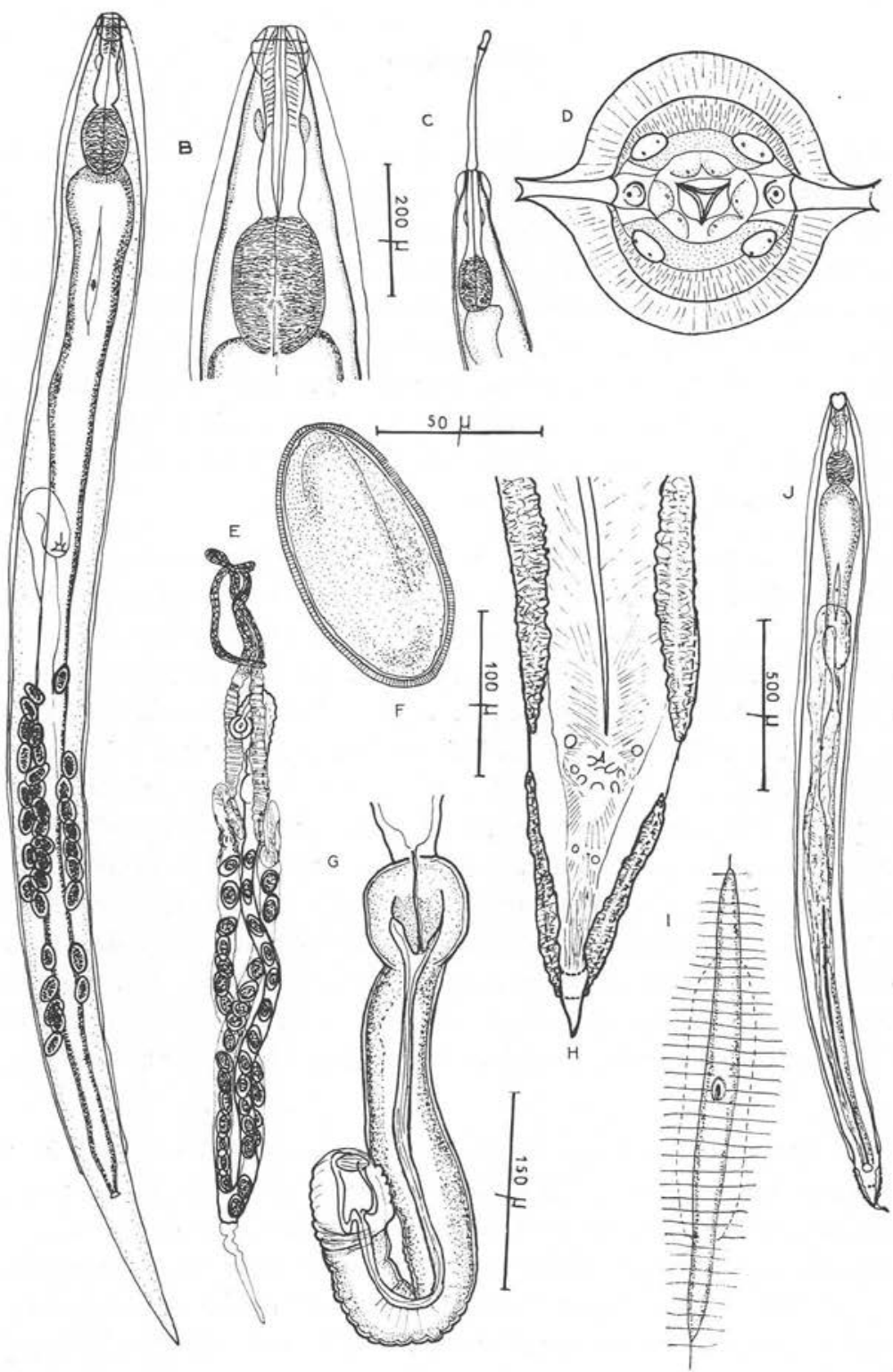

Fig. 3. - Aspiculuris africana n. sp. A) Femelle, vue latérale. B) Extrémité antérieure d'une femelle, vue ventrale. C) Extrémité antérieure, vue latérale, œsophage évaginé. D) Tête, vue apicale. E) Appareil génital femelle. F) Cuf. G) Ovéjecteur. H) Extrémité caudale mâle, vue ventrale. I) Pore excréteur. J) Mâle, vue ventrale. A, C, E, J : éch. $500 u$; B : éch. $200 \mu$; D, H, I : éch. $100 \mu ; \mathrm{F}$ : éch. $50 \mu ; \mathrm{G}$ : éch. $150 \mu$ 


\section{Description}

Oxyures à corps très allongé (fig. $3 \mathrm{~A}$ ) orné latéralement de deux ailes latérales continues, larges chacune de $30 \mu$ au maximum chez la femelle, de $24 \mu$ chez le mâle et s'arrêtant en avant de l'anus. Extrémité céphalique entourée d'une vésicule cuticulaire. Le plateau facial est ovalaire. Il porte en son centre une bouche petite à trois lèvres, une dorsale et deux latéro-ventrales. L'orifice buccal est entouré de deux cycles de papilles, un cycle interne de six petites papilles, un cycle externe de quatre papilles portant chacune deux terminaisons nerveuses. Les amphides sont situées latéralement à la naissance des deux ailes latérales. Chez quelques mâles et femelles gravides l'œsophage s'évagine plus ou moins en une trompe dont l'extrémité porte l'appareil valvulaire normalement situé au fond du bulbe

Mâle (fig. $3 \mathrm{~J}$ ): Spécimen holotype long de $2,5 \mathrm{~mm}$ (les longueurs des différents mâles examinés varient entre 2,3 et $3,6 \mathrm{~mm}$ ) ; largeur au milieu du corps $150 \mu$. Capsule céphalique longue de $65 \mu$, large de $60 \mu$. Anneau nerveux, extrémité postérieure de l'œsophage sans le bulbe, extrémité postérieure du bulbe, situés respectivement à 90 , 170, 280 et $580 \mu$ de l'apex. Largeur du bulbe $80 \mu$. En plus des deux ailes latérales, une arête cuticulaire médiane longue de $800 \mu$ débute ventralement à $950 \mu$ en avant de l'extrémité caudale et se termine à $15 \mu$ du cloaque. Les ailes latérales s'arrêtent à $40 \mu$ du cloaque. Elles sont remplacées par la bourse caudale ornée de quatre renflements cuticulaires: deux renflements préanaux se terminent $15 \mu$ en avant de l'anus, deux postanaux débutent $15 \mu$ en arrière de l'anus (fig. $3 \mathrm{H}$ ). Un espace de $30 \mu$ sépare de chaque côté ces renflements. Pas de spicule. Le cloaque est entouré de quatre paires de papilles: une paire préanale, une para-anale et deux postanales. Une papille double médiane est située en arrière du cloaque, et, entre celui-ci et l'extrémité caudale se situe une autre paire de papilles. Longueur de la queue $165 \mu$; longueur de la pointe caudale $22 \mu$.

Femelle: Le spécimen allotype mesure 4,05 mm de long. (Les longueurs des différentes femelles varient entre 3,2 et $4,2 \mathrm{~mm}$ ). Largeur au niveau de la vulve $260 \mu$. Capsule céphalique longue de $90 \mu$. Anneau nerveux, extrémité postérieure de l'œsophage, extrémité postérieure du bulbe, pore excréteur et vulve situés respectivement à $160,280,490,810 \mu$ et $1,61 \mathrm{~mm}$ de l'apex. Le bulbe est large de $140 \mu$, le pore excréteur débouche au centre d'une plaque sous-cuticulaire très allongée $(280 \times 20 \mu)$. L'appareil génital femelle est constitué de deux branches utérines qui se rejoignent pour former une trompe impaire longue de $1.250 \mu$. Celle-ci se prolonge par un ovéjecteur long de $500 \mu$, large de 50 à $60 \mu$ terminé par un sphincter (fig. $3 \mathrm{E}$ ). Les œufs à coque mince mesurent $87-90 \mu \times 46-42 \mu$. La queue est longue de $480 \mu$ (fig. $3 \mathrm{~F}$ ). 


\section{Discussion}

Cette espèce appartient au genre Aspiculuris Schulz 1924 car les mâles sont dépourvus de spicule. La présence d'un bulbe œsophagien et d'ailes latérales continues et uniformes l'apparente au sous-genre Pseudaspiculuris Akhtar 1955, qui comprend deux espèces :

- Aspiculuris ackerti Kruidenier et Mehra 1959, parasite de Neotoma albigula Hartley et $N$. cinerea Baird en Arizona.

- A. asiatica Schulz 1927 parasite de Rhombomys opimus Licht. au Kazakhstan et au Turkestan.

Nous écartons la première espèce fort différente de nos spécimens par la morphologie de son plateau facial, la faible longueur de ses ailes latérales et la disposition des papilles sur la bourse caudale du mâle.

A. asiatica par contre présente une grande analogie avec notre matériel dans la morphologie des ailes latérales chez le mâle et la femelle et celle de la bourse caudale du mâle.

Cependant, outre les différences de taille (nos spécimens mâles et femelles sont deux fois plus petits que ceux décrits par Schulz), les mâles présentent ventralement en avant du cloaque une arête médiane non spécifiée dans la description très précise de cet auteur. Les œufs sont de dimensions légèrement supérieures, chez nos spécimens $(90 \times 42 \mu)$, à celles d'A. asiatica $(81 \times 37 \mu)$, A. asiatica n'a été signalé qu'au Turkestan et au Kazakhstan.

Nous pensons que notre matériel appartient à une espèce différente de $A$. asiatica. Nous la considérons comme nouvelle et la nommons $A$. africana n. sp.

\section{Résumé}

Cinq espèces d'Oxyures ont été récoltées chez des Muridae africains, deux sont cosmopolites: Syphacia (Syphacia) obveolata (Rud. 1802) et Aspiculuris tetraptera (Nitzsch-1821); trois sont nouvelles, ce sont:

- Syphacia (Syphacia) lophuromyos n. sp. caractérisé par un spicule court (75 $\mu$ ) et par une pointe caudale chez le mâle relativement longue.

- S. (S.) megaloon n. sp. se différencie aisément des autres espèces de Syphacia de Muridae par la très petite taille des femelles dont les œufs sont très volumineux. Le spicule chez le mâle est très court.

- Aspiculuris africana n. sp. se différencie de A. asiatica (Schulz 1927), dont il se rapproche le plus morphologiquement, par une taille deux fois plus faible des mâles et des femelles et des œufs de dimensions légèrement supérieures. 


\section{Bibliographie}

AKHTAR (S. A.), 1955. - On nematode parasite of rats and mice of Lahore, with some remarks on the genus Aspiculuris Schulz 1924 and two new species of the genus. Pakistan J. Sc. res., 7 (3), p. 104-111.

Baylis (H. A.), 1928. - On a collection of nematodes from Nigerian Mammals (chiefly Rodents). Parasit., 20 (3), p. 280-304, fig. 1-25.

BernaRd (J.), 1961. - Quelques espèces d'Helminthes de Micromammifères récoltés en France et en Espagne. Vie et milieu, 12 (1), p. 125-149, fig. 1-2.

Kruidenier (J. F.) et Mehra (K. N.), 1959. - Aspiculuris ackerti n. sp. Nematoda: Oxyuridae, from the wood Rats of Arizona. Proc. Helm. Soc. Washington, 26 (2), p. $147-$ 150, fig. 1-5.

Morgan (D. O.), 1932. - Oxyuris stroma Linstow 1884. Journ. Helminth., 10 (1), p. 15-20, fig. 1-6.

Roman (E.), 1951. - Etude écologique et morphologique sur les Acanthocéphales et les Nématodes parasites des Rats de la région lyonnaise. Mém. Mus. Nat. Hist. Nat., Sér. A, 2, p. 49-270, fig. 1-90 + 2 pl.

Schulz (R. E. S.), 1927. - On the genus Aspiculuris Schulz 1924, and two new species of it, A. dinniki and A. asiatica from Rodents. An. Trop. Med., 21 (2), p. 267-275, fig. 1-7.

Seurat (L. G.), 1916. - Sur les Oxyures de Mammifères. C.R. Soc. Biol., 79, p. 64.

Skrjabin (K. I.), Schikhobalowa (N. P.) et Lagodowskaja (E. A.), 1960. - Osnovi Nematodologi. VIII. Oxyurata, $1^{\text {re }}$ part,, 557 p., 280 fig.

Yamaguti (S.), 1961. - Systema Helminthum. III. The Nematodes of Vertebrates, 2 vol., 1.261 p., $102 \mathrm{pl}$.

[Laboratoire de Zoologie (Vers) (Professeur A.-G. Chabaud).

Muséum National d'Histoire Naturelle] 